\title{
Label-Free Biosensors for the Detection and Quantification of Cardiovascular Risk Markers
}

\author{
Kallempudi S. Saravan, Ozgur Gul, Huveyda Basaga, Ugur Sezerman, and Yasar Gurbuz* \\ Sabanci University, Faculty of Engineering and Natural Sciences, Istanbul 34956, Turkey
}

(Received: xx Xxxx xxxx. Revised/Accepted: $x x$ Xxxx xxxx)

\begin{abstract}
This paper presents a biosensor implementation for the detection of protein molecules using specific antibodies. Affinity sensors allow the detection and quantification of target molecules in complex mixtures by affinity-based interactions. Immobilized antibody molecules are the probes that bind to specific protein molecules (targets) in biological fluids. In this study, inter-digitated electrodes in the form of capacitance on glass slide were designed, fabricated and used to measure the changes in the dielectric properties of the inter-digitated capacitances. Our results in this study present that with a careful design of micro-interdigitated capacitors, a wider dynamic range and higher sensitivity can be achieved for the detection and quantification of C-Reeactive Protein.
\end{abstract}

Keywords: Biosensor, Antibody, Capacitive, Cardiovascular, C-Reactive Protein.

\section{INTRODUCTION}

Biosensors are fast, direct, and with ability to perform label-free measurements are attractive for different applications. The affinity based interactions/sensing approaches by name mean use of specific antigen/antibody, allow the detection and quantification of target molecules in complex mixtures. So, with this approach we can detect the specific proteins without labeling (Label-free) them. Immobilized antibody molecules are the probes that bind to specific protein molecules (targets) in biological fluids. Inter-digitated electrodes in the form of capacitance on glass slide can be used to measure the changes in the dielectric properties of the inter-digitated capacitances upon antigen binding. ${ }^{1}$

Besides the inexpensive production and low sample consumption, main advantages of inter-digitated, capacitive immuno-sensors are (1) the label-free detection mechanisms and (2) the fast and reliable measurements. Unlike other detection mechanisms used to quantify protein concentrations, capacitive method does not need labeling of samples prior to analysis. Upon binding of protein molecules to the immobilized antibody molecules on the surface of electrodes, dielectric properties between the inter-digitated electrodes changes and measurement can be taken immediately after hybridization..$^{2,3}$ The detection mechanism of these sensors is based on the change in

${ }^{*}$ Corresponding author; E-mail: yasar@sabanciuniv.edu the dielectric constant of the inter-digitated capacitance. This change arises, at the simplest form, from the equation $C=\varepsilon \varepsilon_{\mathrm{o}} A / d$, where $C$ is the capacitance between the inter-digitated electrodes, $\varepsilon$ is the dielectric constant of the medium between the plates, $\varepsilon_{\mathrm{o}}$ is the dielectric constant of free space, $A$ is the area of the plates and $d$ is the distance. When a change in dielectric constant occurs, a counter change occurs in capacitance value in between the electrodes. ${ }^{1}$

This change in the dielectric constant, hence in the capacitance, is correlated to the bound antigen molecules to the capture antibodies on the surface, between the electrodes. The dielectric constant of the antibodies changes the dielectric constant between the electrodes/fingers and this result in a change in capacitance. Inter-digitated electrode arrays are used in order to increase the active area for higher sensitivity to binding events. Atomic Force Microscopy (AFM) and electrochemical methods are used to measure this change, but impedance analysis gives faster results. ${ }^{4}$

Biosensor applications for various human serum proteins are available. C-reactive protein (CRP) is one of the inflammation markers in human serum. ${ }^{5}$ Recent articles have shown significance of inflammatory markers in early detection of cardiovascular disease. ${ }^{6,7} \mathrm{CRP}$ is emerging as a new marker for acute phase inflammation and has showed a lot of potential for earlier detection of cardiovascular diseases. ${ }^{8,9}$ 


\section{STRUCTURAL MODELLING AND EXPERIMENTAL}

\subsection{Structural Design and Modeling}

Multi-finger types of capacitors are widely used in microstrip technology. The capacitor itself is defined between the two ports as shown in Figure 1(a). The interdigital capacitor couples the two coplanar waveguides by the electromagnetic field in its region. In a nutshell, the variation of medium between the fingers of inter-digital capacitor, will lead to variation in effective dielectric constant of the area. This directly changes the total capacitance of inter-digital capacitance. Figure 1(b) depicts the equivalent circuit of the inter-digital capacitor. Magnetic coupling between the fingers, a transformer with the selfinductances $L_{1}$ and $L_{2}$ and the mutual inductance $M$ is used. In order to decrease these inductances, long fingers are not being used. The ohmic losses that occur due to the current flow through the fingers can be descried by two frequency dependent resistors $R_{\mathrm{f} 1}$ and $R_{\mathrm{f} 2}$. There resistances must be decreased for instance using thicker metal layer to get rid of losses. The capacitances $C p 1$ and $C p 2$ represent the stray fields from the fingers to the ground plane. Therefore in our design the distance between the ground layer and inter-digital electrodes is increased.

The simulation of this type of structures at high frequencies is not easy and electromagnetic simulators like MOMENTUM/HFSS should be used for high accuracy. In this work, modeling and simulation of inter-digital capacitors are performed using ADS (Advance Design System) MOMENTUM ${ }^{\circledR}$ and HFSS ${ }^{\circledR}$ tools.

The structure/model, shown in Figure 1, is simulated in the frequency range of $1 \mathrm{GHz}$ to $5 \mathrm{GHz}$. One of the ports is grounded during the simulations. The optimum frequency range, selected the one having a maximum capacitance change, is between $2-3 \mathrm{GHz}$. This frequency range is also known as industrial, scientific and medical (ISM) radio bands, originally reserved internationally for noncommercial use of RF electromagnetic fields for industrial, scientific and medical purposes. Figure 2 presents the fabricated/realized inter-digital capacitor structure. (a)

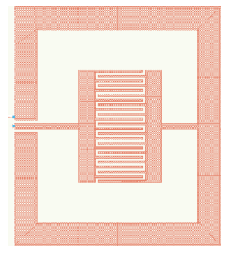

(b)

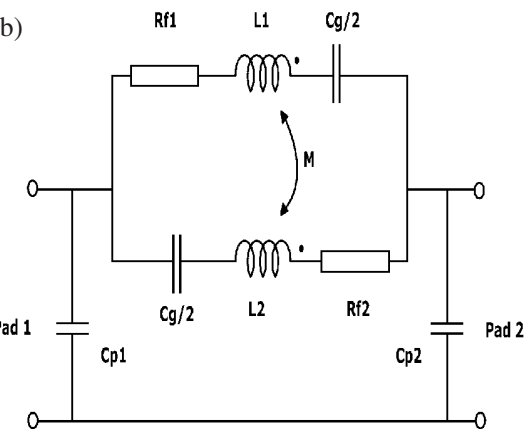

Fig. 1. Two-port, inter-digitated capacitors (a) and its electrical model (b).

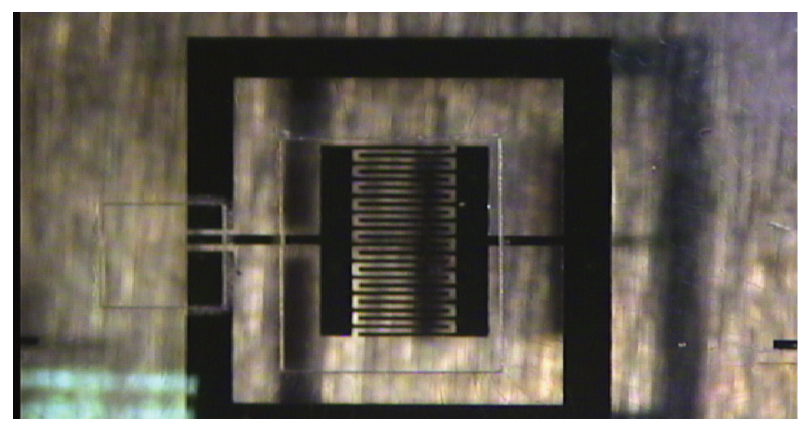

Fig. 2. Nickel IDC with SU8 wells.

The $S$-parameters of the structure in Figure 1 are extracted from the simulation results and actual values are measured using a Network Analyzer. The resulting capacitance values from simulations and measurements for the mentioned frequency range are presented in Figure 3, respectively. The Figure 3 envisages that the capacitance value by simulation at $2.354 \mathrm{GHz}$ is found as $1.829 \mathrm{pF}$, Figure 3(a) that is very close to measured value of $1.8 \mathrm{pF}$, Figure 3(b).

\subsection{Sensor Fabrication}

The fabrication flow for the structure shown in Figure 2 is presented in Figure 4. Very thin tungsten layer of $30 \mathrm{~nm}$ is sputter deposited on the glass microscope slide, which is used to improve the adhesion of gold on substrate. Then $300 \mathrm{~nm}$ of gold is deposited using sputter deposition, as seen in step (A). Following this step, the gold layer is patterned by wet etching with the mask seen in the step (B). $\mathrm{I}_{2} / \mathrm{KI} / \mathrm{H}_{2} \mathrm{O}$ solution is used to etch gold layer and $30 \%$ hydrogen peroxide $\left(\mathrm{H}_{2} \mathrm{O}_{2}\right)$ is used to etch the underlying tungsten layer. After that, an oxide layer of $50 \mathrm{~nm}$ is deposited on the electrodes, step (C), and the top of the contact pads are opened using HF solution, step (D). The oxide layer is deposited to improve the coating of epoxy silane layer, which will be used to immobilize antibodies and to insulate the electrodes. Length of each electrode is $750 \mu \mathrm{m}$ and width is $25 \mu \mathrm{m}$. The distance between two electrodes is $25 \mu \mathrm{m}$. To ease the process of fabrication gold metal is replaced with Nickel. Image Reversal Technique with AZ $5214 \mathrm{E}$ is used to Pattern Nickel Metal, step (D) in the above process is omitted as Nickel readily forms oxide. A $40 \mu \mathrm{m}$ deep SU8 wells were patterned over the inter-digitated structure for easing the antibody immobilization on the sensor structure. Figure 2 shows the fabricated Nickel IDC with SU8 wells.

\subsection{Materials and Reagents}

Monoclonal antibody and purified antigens for C-Reactive Protein were purchased from Fitzgerald Industries International (Concord, MA, USA). Alexa-488 conjugated 
(a)

Eqn $C=11\left(-2^{*}\right.$ piffreq* $^{*}(i m a g($ stoz $(S, 50)))$
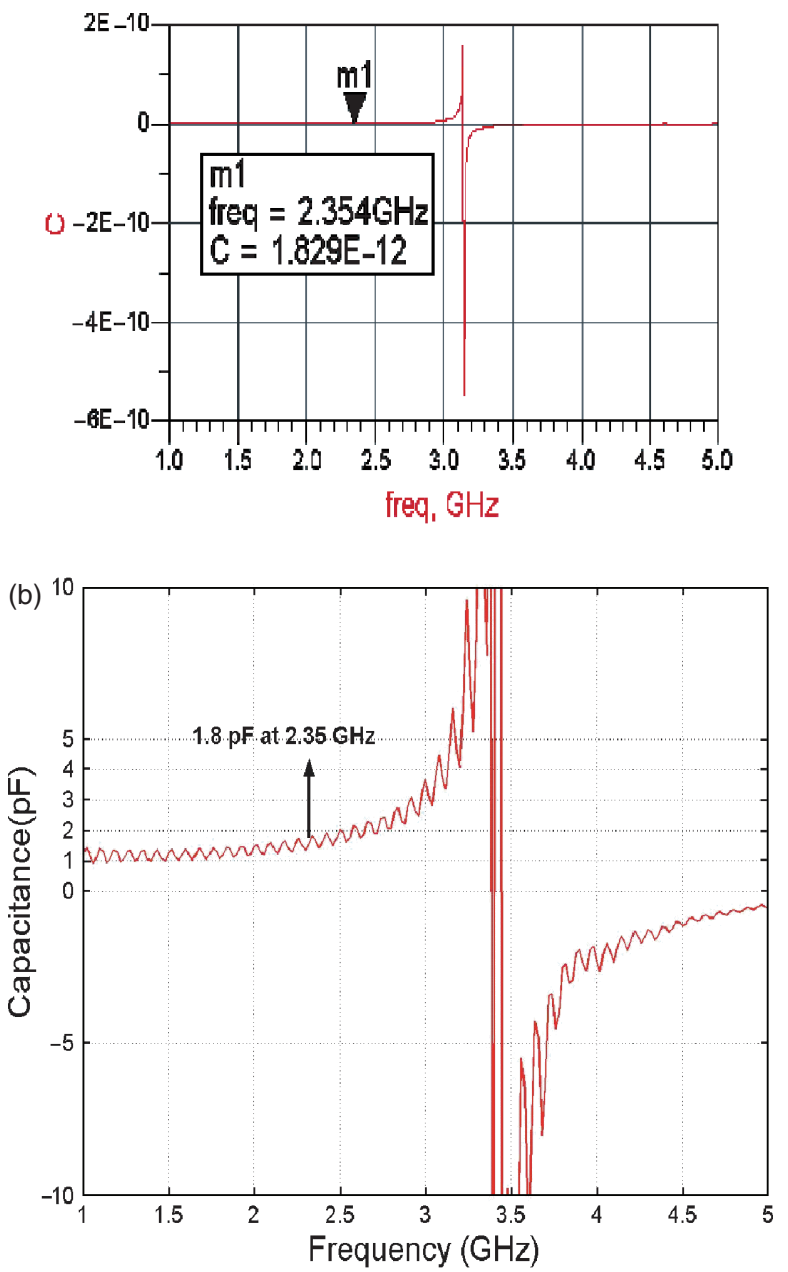

Fig. 3. Simulated (a) and measured (b) values of the inter-digital capacitors.

rabbit anti-mouse antibody was purchased from Invitrogen (Carlsbad, CA, USA). (3-glycidoxypropyl)-trimethoxy silane (GPTS), BSA, PBS and Tween 20 were purchased from Sigma (USA). Fluorescent scanning was carried out using an ArrayWoRx(R) Biochip Reader (Applied Precision, Marlborough, UK) and analyzed by using accompanying software. PBS-T (1X PBS, $0.5 \%$ Tween 20) and Diluent buffer (2\% BSA in BPS-T) were used for all washing and dilution steps, otherwise noted.

\subsection{Surface Activation and Antibody Immobilization}

$2 \%$ of (3-glycidoxypropyl)-trimethoxy silane (GPTS) solution was used to coat $\mathrm{SiO}_{2}$ surface. After one hour silanization reaction, sensors were washed several times with ethanol and dried using centrifuge. $2 \mu \mathrm{l}$ of C-reactive protein antigen at $0.5 \mathrm{mg} / \mathrm{ml}$ concentration was added onto surface and incubated for two hours at room temperature. After immobilization step, biosensor surface was blocked using 2\% BSA for nonspecific binding of antigen. After

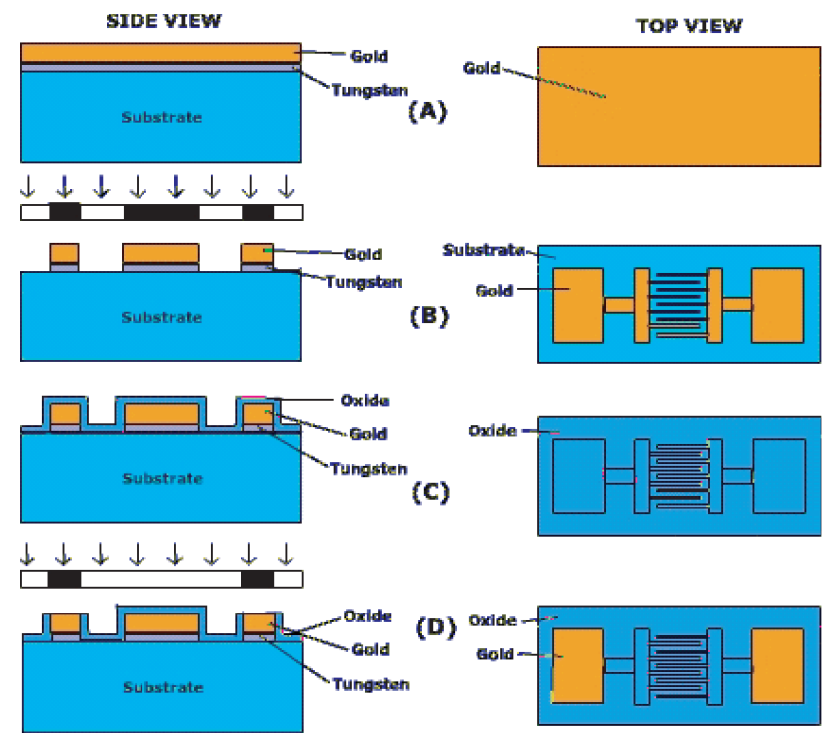

Fig. 4. Inter-digital capacitor fabrication/process.

several times washing with PBS-T, sensors were stored at $4{ }^{\circ} \mathrm{C}$ until use.

\subsection{Measurements}

Purified antigens were diluted in diluent buffer and incubated with sensors for one hour. After several washing steps, sensors were dried using centrifuge. Karl-Suss PM-5 RF Probe Station and Agilent-8720ES S-Parameter Network Analyzer were used for all capacitance measurements. Network Analyzer were calibrated using SOLT (short-open-load-through) method and S-Parameter data of the capacitor were measured. Finally, capacitance values $(C)$ were extracted from the measurements at certain frequencies $(f)$. Only 1 to $5 \mathrm{GHz}$ range was scanned.

\section{RESULTS AND DISCUSSION}

\subsection{Antibody Immobilization}

Immobilization of C-reactive protein specific antibodies onto epoxy coated biosensor surface was checked using Alexa-488 labeled antibodies. Fluorescent conjugated antimouse antibodies were hybridized with capture antibodies on the surface. Fluorescent scanner was used to scan the sensors fabricated on the microscope slide by following the method mentioned in experimental section.

Fluorescent image can be seen in Figure 5. We have found that $0.5 \mathrm{mg} / \mathrm{ml}$ antibody concentration is optimum for immobilization procedure. 2\% BSA blocking is shown to be efficient, as seen in Figure 5, where no fluorescent signal was detected when only BSA blocked sensor was incubated with fluorophore labeled anti-mouse antibody. 

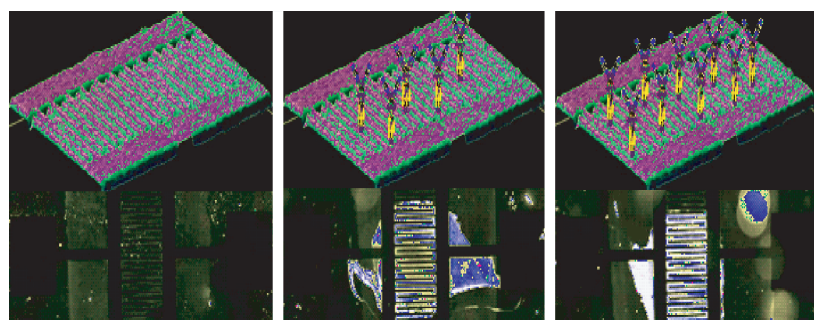

Fig. 5. Antibody immobilization confirmed by hybridization with Alexa-488 labeled anti-mouse antibodies.

\subsection{Antigen Detection}

C-reactive protein antigen were diluted to different concentration using diluent buffer and hybridized with sensor for 1 hour. After several washing steps and drying, capacitance measurements were carried out by following the protocol in experimental section. Capacitance values were extracted from measurements for each different antigen concentration. We have observed an inductive behavior after $2.7 \mathrm{GHz}$ frequency, therefore capacitance values for frequencies higher than $2.7 \mathrm{GHz}$ were not considered. Capacitance change versus frequency graph can be seen in Figure 6.

Concentrations versus capacitance changes were calculated for some of the frequency points, as shown in Figure 7(a) and changes in capacitance at various stages blank(0), epoxy coated(1), antibody coated(2), blocked(3), and antigen incubation(4), were presented in Figures 7(b) and (c). These frequencies can be used for further experiments. We have found that concentration versus capacitance change at $2.62 \mathrm{GHz}$ is linear over $100 \mathrm{ng} / \mathrm{ml}$ to $800 \mathrm{ng} / \mathrm{ml}$ antigen concentration range, with $R^{2}$ equals to 0.97, as presented in Figure 8.

We have showed that capacitance change upon binding of antigen to the immobilized capture antibodies on the surface is correlated to the antigen concentration in the buffer. Although the detection range is not low comparing

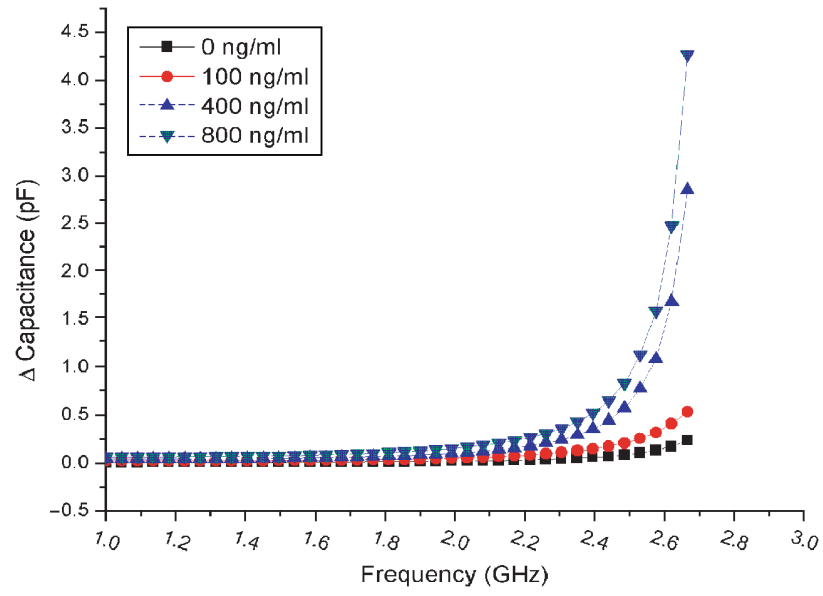

Fig. 6. Frequency versus capacitance change for different antigen concentration.

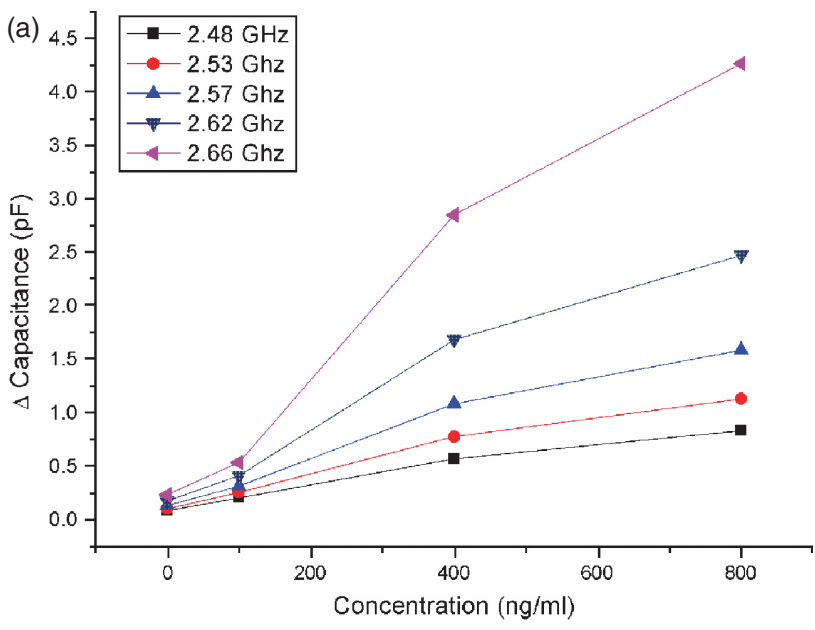

(b)

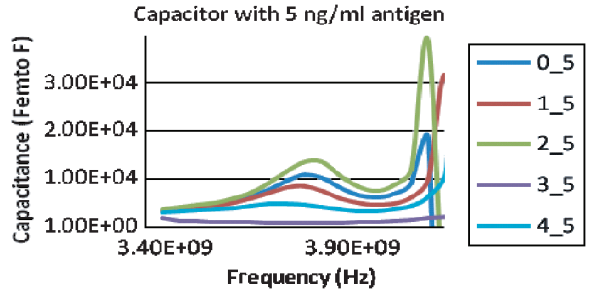

(c) $3.00 \mathrm{E}+04 \quad$ Capacitor with $25 \mathrm{ng} / \mathrm{ml}$ of antigen

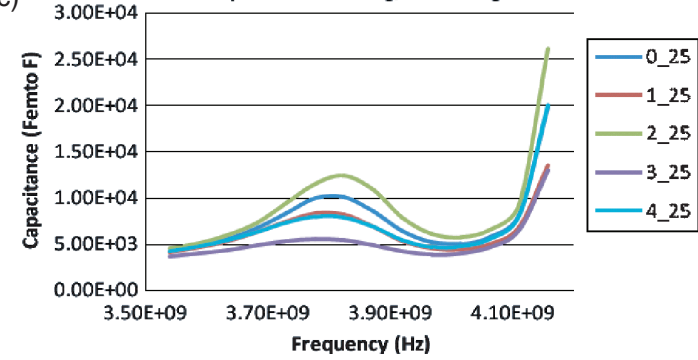

Fig. 7. Concentration versus capacitance change at different frequencies (1-25 means Capacitance measured after epoxy coating that will be incubated with $25 \mathrm{ng} / \mathrm{ml}$ antigens).

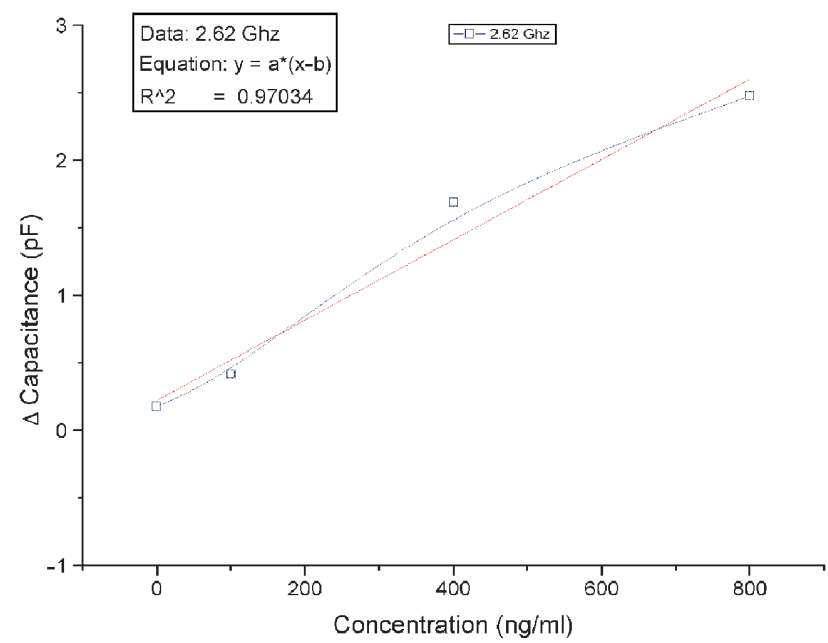

Fig. 8. Capacitance change versus antigen concentration at $2.62 \mathrm{GHz}$. 
to other available systems, optimization of capacitor geometries can further enhance the sensor detection limits.

\section{CONCLUSIONS}

In this study, we have analyzed the reliability of interdigitated electrodes for biosensor applications. Biosensors based on antibody-antigen interactions can be used for quantification of biomarkers in human serum. High sensitivity and improved detection range can be obtained through optimization of topology/geometry of capacitors. Furthermore, integration with microfluidics systems for sample delivery can further improve the overall stability and reproducibility of inter-digitated electrodes based immuno-biosensor.

Although we have used C-reactive protein as a model system, this antibody based, label-free detection system can be applied to other areas, such as, microbiological detection, DNA detection or biomarker detection.

Inter-digitated electrodes can be further designed in an array format and integrated into other Micro-ElectroMechanical devices/systems for further improvements of performance, cost, reliability, etc. Using arrays of capacitors may allow us to quantify more than one marker at a time and enhance the diagnostic power of biosensor.
Besides high throughput capability of these biosensors, further integration of other electronics parts can improve the signal-to-noise ratio as well.

Acknowledgment: We thank Bulent Koroglu for his valuable contribution to the processing of devices. We also thank the Scientific and Technological Research Council of Turkey (TÜBİTAK) for the financial support for this project under the contract number 107E014 and title "RF Transmitter-Based Transducer for Biosensor Applications."

\section{References and Notes}

1. C. Bergren, Electroanalysis 13, 173 (2001).

2. M. DeSilva, Y. Zhang, P. Hesketh, G. Maclay, S. Gendel, and J. Stetter, Biosensors Bioelectron. 10, 675 (1995).

3. A. R. Varlan, J. Suls, W. Sansen, D. Veelaert, and De. Loof, Sens. Actuators, B 44, 334 (1997).

4. G. Zeng and Z. Zheng, Proteomics 5, 4347 (2005).

5. M. P. De Maat and A. Trion, Curr. Opin. Lipidol. 15, 651 (2004).

6. P. M. Ridker, Circulation 108, e81-5 (2003).

7. V. B. Patel, M. A. Robbins, and E. J. Topol, Cleve Clin. J. Med. 68, $521(2001)$

8. M. B. Clearfield, J. Am. Osteopath. Assoc. 105, 409 (2005).

9. F. Lombardi, F. Tundo, P. Terranova, P. M. Battezzati, M. Ramella, A. Bestetti, and L. Tagliabue, Int. J. Cardiology 98, 313, (2005). 